\title{
Analysis of Indoor Radon Level and its Health Risks Parameters in Three Selected Towns in Port Harcourt, Rivers State, Nigeria
}

\author{
Orlunta Aloysius Ndubisi a,*, Briggs-Kamara Margaret Apaemi ${ }^{\mathrm{a}}$, Sigalo Friday Barikpe ${ }^{\mathrm{a}}$, Iyeneomie \\ Tamunobereton-Ari ${ }^{\mathrm{a}}$
}

${ }^{a}$ Department of Physics, Faculty of Science, Rivers State University, Nkpolu Oroworukwo, Port Harcourt, Rivers State, Nigeria.

\begin{abstract}
Analysis of indoor radon level and its health risk parameters has been carried out in Borikiri (BT), Diobu (DR), and Rebisi (RB) towns in Port Harcourt, Rivers State, Nigeria. A pocket sized Corentium Arthings digital radon detector meter was used to record the indoor radon concentration levels. The geographical coordinates were recorded using a hand-held geographical positioning system (GPS) for the various sample points. A total of ten houses were measured for each town making a total of 30 sample points for the three communities. The results obtained show that for Borikiri town, the concentration level varied from $30.7100-19.9800 \mathrm{Bqm}^{-3}$ with an average of $11.32 \pm 2.59 \mathrm{Bqm}^{-3}$. The annual absorbed dose varied from $7.7478-1.1202 \mathrm{mSv} / \mathrm{yr}$ with a mean value of $2.59 \pm 0.65 \mathrm{mSv} / \mathrm{yr}$ while the annual equivalent dose rate varied from $0.829-0.336$ $\mathrm{mSv} / \mathrm{yr}$ with an average of $0.69 \pm 0.16 \mathrm{mSv} / \mathrm{yr}$ The excess life time cancer risk calculated for seventy years $(70 \mathrm{yrs})$ varied from $6.510-0.941 \mathrm{with}$ an average of $2.45 \pm 1.71$. The results of the indoor concentration level for Diobu town ranged from $37.74-5.9200 \mathrm{Bqm}^{-3} \mathrm{with}^{\mathrm{a}} \mathrm{mean}$ value of $12.95 \pm 2.91 \mathrm{Bqm}^{-3}$. The annual absorbed dose for the area ranged from $9.5214-1.1494$ with an average of $3.26 \pm 0.73 \mathrm{mSv} / \mathrm{yr}$, the annual equivalent dose rate varied from $0.694-0.359$ with a mean of $0.78 \pm 0.8$, the excess life time cancer risk calculated for seventy years ranged from $8.000-1.725$ with a mean of $2.91 \pm 0.61$. The indoor concentration level for Rebisi town ranged from $12.9500-4.0700 \mathrm{Bqm} \mathrm{m}^{-3} \mathrm{with}$ an average of $8.55 \pm 1.00$, the annual absorbed dose ranged from $3.2671-1.0268 \mathrm{mSv} / \mathrm{yr}$, the annual equivalent dose rate varied from $0.784-0.269 \mathrm{with}$ an average of $0.52 \pm 0.06$, the excess life time cancer risk of $2.745-0.863$ with an average of $1.82 \pm 0.21$. The results of the indoor concentration levels, the annual absorbed dose and the annual effective dose rate are all below the ICRP safe limit. However, the results of the excess life time cancer risk are all higher than the ICRP safe standard limit of $0.029 \times 10^{-3}$.
\end{abstract}

DOI:10.46481/jnsps.2021.203

Keywords: indoor radon, annual absorbed dose, annual effective dose, excess life cancer risk

Article History :

Received: 20 April 2021

Received in revised form: 10 May 2021

Accepted for publication: 09 July 2021

Published: 29 August 2021

(C)2021 Journal of the Nigerian Society of Physical Sciences. All rights reserved. Communicated by: W. A. Yahya

\section{Introduction}

Radon is a radioactive gas which is colourless, odourless and tasteless. It is predominantly found in rock samples, bedrock formations, soil and ground water all over the world, [1]. Radon222 is the immediate decay product of radium- 226 during the

${ }^{*}$ Corresponding author tel. no: $+234(0) 8060700503$

Email address: aloyndubisi@gmail . com (Orlunta Aloysius Ndubisi ) decay series of Uranium-238 which is the source of radon-222. It has a half-life of 3.81 days. Radon-222 decays to polonium298 with the release of an alpha particle and consequently to other progenies of radon or radon daughters. Radon has been classified by the international Agency for Research on cancer [2] as a carcinogenic gas.

The World Health Organization [3] also classify radon as 
the second highest cause of lung cancer after cigarette smoking. It was estimated that radon-222 causes between 3\% to $14 \%$ of all lung cancers. Radon is the leading cause of lung cancer among non-smokers. It is the cause of about fifteen percent of all lung cancer cases throughout the world [4]. People are exposed to high radon level in small houses than in bigger apartments [5]. The entry routes of indoor into the houses includes, the door, the windows, cracks on the walls, sinks, basements, floors etc.

The health effects as a result of higher exposure to radon222 , is radiation induced. Inhalation is the main route through which humans are exposure to radon radiation. The dose contribution to radon may be small, but the inhalation of the progenies of radon which have very short half-life can deposit nonhomogenously in the human respiratory track and irradiate the bronchial epithelium which is usually very harmful.

Two of the radon progenies (daughters) Polonium-214 and Polonium-218 release the highest amount of alpha radiation dose to the lungs [6]. When these radioactive particles settle in a person's lung they can cause damage to the mucosa linings of the lungs. A prolonged exposure to radon-222 radiation can also lead to series of damage to the pulmonary mucosa which could result to lung cancer.

An individual's average radiation come from the decay products of radon-222 such as, polonium-218 and polonium-214. These products of radon-222 are in the solid form. They could be attached or unattached to the surface of aerosols, dusts and smoke particles. They can adhere deeply or stick to the lungs where they irradiate and penetrate the cells of the mucosa membranes, bronchi and pulmonary tissues. The ionizing radiation which emanate from the radioactive decay of radon-222 begins the process of carcinogenesis in the human lungs.

It is estimated that about $90 \%$ of the radon progenies can attach to airborne particles [7]. Also, the unattached fraction of the particles which constitutes about $10 \%$ has a higher rate of deposition and is more efficient in delivering doses to the sensitive cells of the lungs [8]. The more the concentration of radon in homes the greater the risk of lung cancer due to radon exposure. The risk of lung cancer increases for every $100 \mathrm{~Bq} / \mathrm{m} 3$ increase in radon concentration, according to the World Health Organization (WHO, 2016). However, there is no thresholds value below which radon exposure carries no risk of lung cancer. Therefore it is important that every country should set up a national reference level "AS Low As Reasonably Achievable" ALARA [9].

The reason for carrying out radon measurements in these towns is because radon-222 concentration level has not been done within Port Harcourt local government. Therefore, we wanted to find out the radon-222 concentration level and compare the results with international set standards. Also, measurement of radon-222 concentration level in these areas will provide baseline studies and literature for other researchers. It is well known fact that these areas are densely populated with building styles that are poorly ventilated therefore, it was necessary to find out if the residents of these towns are exposed to higher dose of radon-222 from their environment. Additionally, there has been incident of lung cancer cases within these areas in recent times even though not officially reported, therefore it was important to confirm if it was as a result of high level of radon exposure.

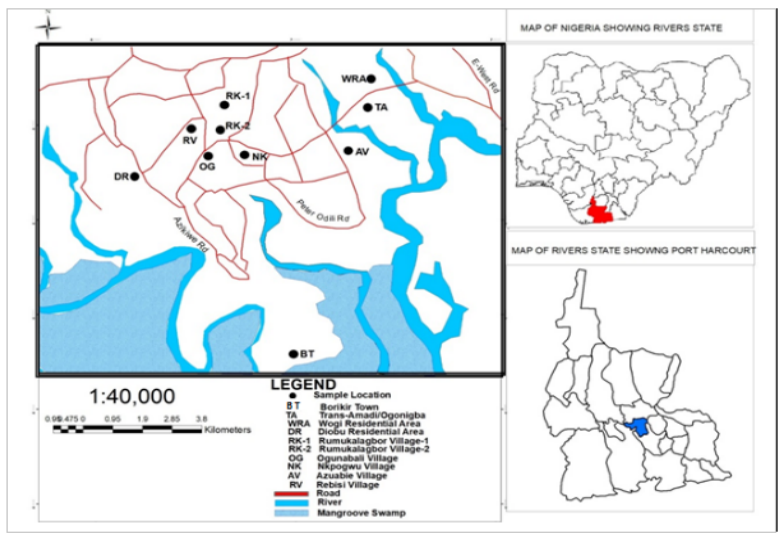

Figure 1. Map of study area

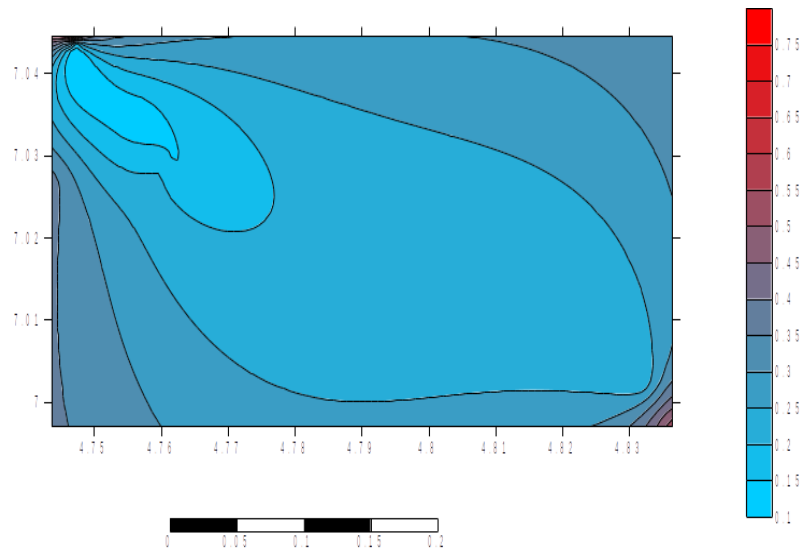

Figure 2. Contour map of Borikiri Town

\section{Materials and Methods}

The radon detector used in this work is Airthings Corentium Digital Radon Detector designed in Oslo Norway in the year 2019. It is calibrated to measure radon-222 in picocurie per litre ( $\mathrm{pCi} / \mathrm{L})$. The Airthings Corentium digital detector can be used to monitor radon concentration levels for a minimum period of 48 hours, for daily, weekly, monthly and yearly. The radon meter works on the principle that the Radon diffuses into a detection chamber. As the atom decay they emit energetic alpha particles. The energetic alpha particles are detected by a silicon photodiode. The alpha particle generates a small signal current when it hits the photodiode. By the use of a low power 
Table 1. Radon Levels in Borikiri Town (BT) and the Geographical Coordinates of the Sample Points.

\begin{tabular}{ccccc}
\hline \hline S/N & $\begin{array}{c}\text { Location } \\
\text { Borikiri Town (BT) }\end{array}$ & $\begin{array}{c}\text { GPS Coordinates } \\
\text { Latitudes }\end{array}$ & Longitudes & Radon Levels (pCi/L) \\
\hline \hline 1.0 & $B T_{1}$ & $N 4^{\circ} 49^{\prime} 55.09524^{\prime \prime}$ & $E 7^{\circ} 0^{\prime} 8.298720^{\prime \prime}$ & 0.21 \\
2.0 & $B T_{2}$ & $N 4^{\circ} 44^{\prime} 50.30664^{\prime \prime}$ & $E 7^{\circ} 2^{\prime} 37.05684^{\prime \prime}$ & 0.18 \\
3.0 & $B T_{3}$ & $N 4^{\circ} 44^{\prime} 48.28200^{\prime \prime}$ & $E 7^{\circ} 2^{\prime} 40.37400^{\prime \prime}$ & 0.83 \\
4.0 & $B T_{4}$ & $N 4^{\circ} 50^{\prime} 11.16600^{\prime \prime}$ & $E 6^{\circ} 59^{\prime} 49.2420^{\prime \prime}$ & 0.54 \\
5.0 & $B T_{5}$ & $N 4^{\circ} 45^{\prime} 24.35400^{\prime \prime}$ & $E 7^{\circ} 2^{\prime} 3.384000^{\prime \prime}$ & 0.12 \\
6.0 & $B T_{6}$ & $N 4^{\circ} 44^{\prime} 47.92800^{\prime \prime}$ & $E 7^{\circ} 2^{\prime} 36.49200^{\prime \prime}$ & 0.24 \\
7.0 & $B T_{7}$ & $N 4^{\circ} 44^{\prime} 45.58200^{\prime \prime}$ & $E 7^{\circ} 2^{\prime} 36.76800^{\prime \prime}$ & 0.27 \\
8.0 & $B T_{8}$ & $N 4^{\circ} 45^{\prime} 41.24400^{\prime \prime}$ & $E 7^{\circ} 1^{\prime} 42.47400^{\prime \prime}$ & 0.15 \\
9.0 & $B T_{9}$ & $N 4^{\circ} 45^{\prime} 34.58400^{\prime \prime}$ & $E 7^{\circ} 1^{\prime} 38.63400^{\prime \prime}$ & 0.21 \\
10.0 & $B T_{10}$ & $N 4^{\circ} 44^{\prime} 37.01838^{\prime \prime}$ & $E 7^{\circ} 1^{\prime} 37.78428^{\prime \prime}$ & 0.37 \\
\hline \hline
\end{tabular}

Table 2. Radon Levels in Diobu Residential Area (DR) and the Geographical Coordinates of the Sample Points.

\begin{tabular}{ccccc}
\hline \hline S/N & $\begin{array}{c}\text { Location } \\
\text { Diobu Residential Area (DR) }\end{array}$ & $\begin{array}{c}\text { GPS Coordinates } \\
\text { Latitudes }\end{array}$ & Longitudes & Radon Levels (pCi/L) \\
\hline \hline 1.0 & $D R_{1}$ & $N 4^{\circ} 47^{\prime} 55.956^{\prime \prime}$ & $E 6^{\circ} 59^{\prime} 22.740^{\prime \prime}$ & 0.22 \\
2.0 & $D R_{2}$ & $N 4^{\circ} 49^{\prime} 9.1560^{\prime \prime}$ & $E 6^{\circ} 59^{\prime} 32.280^{\prime \prime}$ & 0.31 \\
3.0 & $D R_{3}$ & $N 4^{\circ} 48^{\prime} 4.5550^{\prime \prime}$ & $E 6^{\circ} 59^{\prime} 15.906^{\prime \prime}$ & 0.16 \\
4.0 & $D R_{4}$ & $N 4^{\circ} 54^{\prime} 58.524^{\prime \prime}$ & $E 6^{\circ} 59^{\prime} 49.692^{\prime \prime}$ & 0.27 \\
5.0 & $D R_{5}$ & $N 4^{\circ} 55^{\prime} 8.3860^{\prime \prime}$ & $E 6^{\circ} 59^{\prime} 44.119^{\prime \prime}$ & 1.02 \\
6.0 & $D R_{6}$ & $N 4^{\circ} 48^{\prime} 20.874^{\prime \prime}$ & $E 6^{\circ} 59^{\prime} 16.542^{\prime \prime}$ & 0.31 \\
7.0 & $D R_{7}$ & $N 4^{\circ} 47^{\prime} 33.690^{\prime \prime}$ & $E 6^{\circ} 59^{\prime} 32.598^{\prime \prime}$ & 0.24 \\
8.0 & $D R_{8}$ & $N 4^{\circ} 47^{\prime} 35.592^{\prime \prime}$ & $E 6^{\circ} 59^{\prime} 31.740^{\prime \prime}$ & 0.46 \\
9.0 & $D R_{9}$ & $N 4^{\circ} 48^{\prime} 18.100^{\prime \prime}$ & $E 6^{\circ} 59^{\prime} 19.500^{\prime \prime}$ & 0.29 \\
10.0 & $D R_{10}$ & $N 4^{\circ} 48^{\prime} 35.7282^{\prime \prime}$ & $E 6^{\circ} 59^{\prime} 16.11168^{\prime \prime}$ & 0.43 \\
\hline \hline
\end{tabular}

Table 3. Radon Levels in Rebesi Town (RB) and the Geographical Coordinates of the Sample Points.

\begin{tabular}{|c|c|c|c|c|}
\hline $\mathrm{S} / \mathrm{N}$ & $\begin{array}{c}\text { Location } \\
\text { Rebesi Town (RB) }\end{array}$ & $\begin{array}{l}\text { GPS Coordinates } \\
\text { Latitudes }\end{array}$ & Longitudes & Radon Levels (pCi/L) \\
\hline 1.0 & $R B_{1}$ & $N 4^{\circ} 49^{\prime} 19.53522^{\prime \prime}$ & $E 6^{\circ} 57^{\prime} 36.53706^{\prime \prime}$ & 0.35 \\
\hline 2.0 & $R B_{2}$ & $N 4^{\circ} 49^{\prime} 13.3944^{\prime \prime}$ & $E 6^{\circ} 57^{\prime} 32.43258^{\prime \prime}$ & 0.19 \\
\hline 3.0 & $R B_{3}$ & $N 4^{\circ} 49^{\prime} 12.84108^{\prime \prime}$ & $E 6^{\circ} 57^{\prime} 33.7956^{\prime \prime}$ & 0.27 \\
\hline 4.0 & $R B_{4}$ & $N 4^{\circ} 49^{\prime} 7.63932^{\prime \prime}$ & $E 6^{\circ} 57^{\prime} 29.95014^{\prime \prime}$ & 0.16 \\
\hline 5.0 & $R B_{5}$ & $N 4^{\circ} 49^{\prime} 9.0387^{\prime \prime}$ & $E 6^{\circ} 57^{\prime} 24.7416^{\prime \prime}$ & 0.31 \\
\hline 6.0 & $R B_{6}$ & $N 4^{\circ} 49^{\prime} 2.71242^{\prime \prime}$ & $E 6^{\circ} 57^{\prime} 22.00974^{\prime \prime}$ & 0.12 \\
\hline 7.0 & $R B_{7}$ & $N 4^{\circ} 49^{\prime} 9.59052^{\prime \prime}$ & $E 6^{\circ} 57^{\prime} 17.23656^{\prime \prime}$ & 0.24 \\
\hline 8.0 & $R B_{8}$ & $N 4^{\circ} 49^{\prime} 4.3086^{\prime \prime}$ & $E 6^{\circ} 57^{\prime} 57.75876^{\prime \prime}$ & 0.33 \\
\hline 9.0 & $R B_{9}$ & $N 4^{\circ} 49^{\prime} 3.9673^{\prime \prime}$ & $E 6^{\circ} 57^{\prime} 25.41666^{\prime \prime}$ & 0.11 \\
\hline 10.0 & $R B_{10}$ & $N 4^{\circ} 49^{\prime} 7.37353^{\prime \prime}$ & $E 6^{\circ} 57^{\prime} 41.99568^{\prime \prime}$ & 0.23 \\
\hline
\end{tabular}

amplifier stage, the signal current is converted into a large voltage signal. The maximum amplitude of the voltage signal is detected and sampled by an analogue to digital converter (ADC). The amplitude of this signal is proportional to the energy of the alpha particle that hit the photodiode. The brain of the monitor is a micro-controller which registers the time and the energy of every detected particle. This information is used to calculate the mean Radon concentration for, daily, weekly, monthly and yearly periods.
Indoor radon concentration measurement was carried out in three towns in Port Harcourt using the Arthings digital radon detector while the geographical position system (GPS) was used for the measurement of the geographical coordinates of the sample points. A total of 30 sample points were measured for the three towns, Borikiri (BTA) ,Diobu (DR) and Rebisi (RB) making 10 samples each.

The Arthings Corentium digital radon detector was placed in the dwelling room at a height of about $50 \mathrm{~cm}$ above the ground 
Table 4. Computed values of annual effective dose and annual equivalent dose rate of Borikiri Town

\begin{tabular}{lllllll}
\hline $\mathrm{S} / \mathrm{N}$ & $\mathrm{S} / \mathrm{Pts}$ & $\mathrm{C}_{R N}(\mathrm{PCi} / \mathrm{L})$ & $\begin{array}{l}\mathrm{C}_{R N} \\
\left(B q / \mathrm{m}^{3}\right)\end{array}$ & $\begin{array}{l}\mathrm{D}_{R n} \\
(\mathrm{mSv} / \mathrm{y})\end{array}$ & $\begin{array}{l}\text { AEDR } \\
(\mathrm{mSv} / \mathrm{y})\end{array}$ & ELCR $\times 10^{-3}$ \\
\hline 1.0 & $\mathrm{BT}_{1}$ & 0.21 & 7.7700 & 1.9603 & 0.470 & 1.6470 \\
2.0 & $\mathrm{BT}_{2}$ & 0.18 & 6.6600 & 1.6802 & 0.403 & 1.4117 \\
3.0 & $\mathrm{BT}_{3}$ & 0.83 & 30.7100 & 7.7478 & 1.859 & 6.5096 \\
4.0 & $\mathrm{BT}_{4}$ & 0.54 & 19.9800 & 5.0407 & 1.210 & 4.2351 \\
5.0 & $\mathrm{BT}_{5}$ & 0.12 & 4.4400 & 1.1202 & 0.269 & 0.9411 \\
6.0 & $\mathrm{BT}_{6}$ & 0.24 & 8.8800 & 2.2403 & 0.538 & 1.8823 \\
7.0 & $\mathrm{BT}_{7}$ & 0.27 & 5.5500 & 1.4002 & 0.336 & 1.1764 \\
8.0 & $\mathrm{BT}_{8}$ & 0.15 & 7.7700 & 1.9603 & 0.470 & 1.6470 \\
9.0 & $\mathrm{BT}_{9}$ & 0.21 & 13.6900 & 3.4538 & 0.829 & 2.9019 \\
10.0 & $\mathrm{BT}_{10}$ & 0.37 & 7.7700 & 1.9603 & 0.470 & 1.6470 \\
\hline $\mathrm{AVERAGE}$ & $0.31 \pm 0.07$ & $11.32 \pm 2.59$ & $2.59 \pm 0.65$ & $0.69 \pm 0.16$ & $2.40 \pm 0.55$ \\
\hline \hline
\end{tabular}

Table 5. Computed values of excess lifetime cancer risk for ages of ; 70(Std), 60, 50, 40 and 30 yrs of Borikiri Town

\begin{tabular}{lllllll}
\hline $\mathrm{S} / \mathrm{N}$ & $\mathrm{S} / \mathrm{Pts}$ & $\mathrm{ELCR} \times 10^{-3}$ & ELCR $\times 10^{-3}$ & ELCR $\times 10^{-3}$ & ELCR $\times 10^{-3}$ & ELCR $\times 10^{-3}$ \\
& & $70 \mathrm{yrs}(\mathrm{Std})$ & $60 \mathrm{yrs}$ & $50 \mathrm{yrs}$ & $40 \mathrm{yrs}$ & $30 \mathrm{yrs}$ \\
\hline 1.0 & $B T_{1}$ & 1.647 & 1.411 & 1.176 & 0.941 & 0.706 \\
2.0 & $B T_{2}$ & 1.412 & 1.210 & 1.008 & 0.807 & 0.605 \\
3.0 & $B T_{3}$ & 6.510 & 5.578 & 4.649 & 3.719 & 2.789 \\
4.0 & $B T_{4}$ & 4.235 & 3.629 & 3.024 & 2.420 & 1.815 \\
5.0 & $B T_{5}$ & 0.941 & 0.807 & 0.672 & 0.538 & 0.403 \\
6.0 & $B T_{6}$ & 1.882 & 1.613 & 1.344 & 1.075 & 0.807 \\
7.0 & $B T_{7}$ & 2.118 & 1.815 & 1.512 & 1.210 & 0.907 \\
8.0 & $B T_{8}$ & 1.176 & 1.008 & 0.840 & 0.672 & 0.504 \\
9.0 & $B T_{9}$ & 1.647 & 1.411 & 1.176 & 0.941 & 0.706 \\
10.0 & $B T_{10}$ & 2.902 & 2.487 & 2.072 & 1.658 & 1.243 \\
\hline AVERAGE & $2.45 \pm 1.71$ & $2.10 \pm 1.47$ & $1.75 \pm 1.22$ & $1.40 \pm 0.98$ & $1.05 \pm 0.73$ \\
\hline
\end{tabular}

Table 6. Computed values of, annual effective dose and annual equivalent dose rate and risk of Diobu Residential Area (DR)

\begin{tabular}{llllll}
\hline $\mathrm{S} / \mathrm{N}$ & $\mathrm{S} / \mathrm{Pts}$ & $C_{R N}(\mathrm{PCi} / \mathrm{L})$ & $\begin{array}{l}C_{R N} \\
\left(B q / \mathrm{m}^{3}\right)\end{array}$ & $\begin{array}{l}D_{R n} \\
(\mathrm{mSv} / \mathrm{y})\end{array}$ & $\begin{array}{l}\text { AEDR } \\
(\mathrm{mSv} / \mathrm{y})\end{array}$ \\
\hline 1.0 & $D R_{1}$ & 0.22 & 8.1400 & 2.0536 & 0.493 \\
2.0 & $D R_{2}$ & 0.31 & 11.4700 & 2.8937 & 0.694 \\
3.0 & $D R_{3}$ & 0.16 & 5.9200 & 1.4935 & 0.358 \\
4.0 & $D R_{4}$ & 0.27 & 9.9900 & 2.5204 & 0.605 \\
5.0 & $D R_{5}$ & 1.02 & 37.7400 & 9.5214 & 2.285 \\
6.0 & $D R_{6}$ & 0.31 & 11.4700 & 2.8937 & 0.694 \\
7.0 & $D R_{7}$ & 0.24 & 8.8800 & 2.2403 & 0.538 \\
8.0 & $D R_{8}$ & 0.46 & 17.0200 & 4.2939 & 1.031 \\
9.0 & $D R_{9}$ & 0.29 & 10.7300 & 2.7071 & 0.650 \\
10.0 & $D R_{10}$ & 0.43 & 8.1400 & 2.0536 & 0.493 \\
AVERAGE & $0.37 \pm 0.08$ & $12.95 \pm 2.91$ & $3.26 \pm 0.73$ & $0.78 \pm 0.78$ \\
\hline
\end{tabular}

and about $150 \mathrm{~cm}$ from both the window and door and $25 \mathrm{~cm}$ from the walls, [10] The detector was kept for a period of two days (48hrs.) in a given dwelling before relocating to another house or home.

The windows and doors were kept closed throughout the period of the measurement to ensure that the indoor air is not distorted to achieve accuracy within the period of 48 hours.

\subsection{Radon Risk Parameters}

2.1.1. Annual Absorbed dose(DRn) from Radon Concentration

The annual absorb dose calculated from the radon concentration is the magnitude of energy delivered in a tissue from exposure to ionizing radiation within a specified period. The physics of the equation employed in the computation of the annual absorbed dose rate according to the report of United Nation's Scientific Committee on the Effects of Atomic Radiation 
Table 7. Computed values of excess lifetime cancer risk for ages of; 70(Std), 60, 50, 40 and $30 \mathrm{yrs}$ of Diobu Residential Area (DR)

\begin{tabular}{|c|c|c|c|c|c|c|}
\hline $\mathrm{S} / \mathrm{N}$ & $\mathrm{S} / \mathrm{Pts}$ & $\begin{array}{l}\text { ELCR } \times 10^{-3} \\
70 \mathrm{yrs}(\mathrm{Std})\end{array}$ & $\begin{array}{l}\text { ELCR } \times 10^{-3} \\
60 \mathrm{yrs}\end{array}$ & $\begin{array}{l}\text { ELCR } \times 10^{-3} \\
50 \mathrm{yrs}\end{array}$ & $\begin{array}{l}\text { ELCR } \times 10^{-3} \\
40 \mathrm{yrs}\end{array}$ & $\begin{array}{l}\text { ELCR } \times 10^{-3} \\
30 \mathrm{yrs}\end{array}$ \\
\hline 1.0 & $\mathrm{DR}_{1}$ & 1.725 & 1.479 & 1.232 & 0.986 & 0.739 \\
\hline 2.0 & $\mathrm{DR}_{2}$ & 2.431 & 2.083 & 1.736 & 1.389 & 1.042 \\
\hline 3.0 & $\mathrm{DR}_{3}$ & 1.255 & 1.075 & 0.896 & 0.717 & 0.538 \\
\hline 4.0 & $\mathrm{DR}_{4}$ & 2.118 & 1.815 & 1.512 & 1.210 & 0.907 \\
\hline 5.0 & $\mathrm{DR}_{5}$ & 8.000 & 6.855 & 5.713 & 4.570 & 3.428 \\
\hline 6.0 & $\mathrm{DR}_{6}$ & 2.431 & 2.083 & 1.736 & 1.389 & 1.042 \\
\hline 7.0 & $\mathrm{DR}_{7}$ & 1.882 & 1.613 & 1.344 & 1.075 & 0.807 \\
\hline 8.0 & $\mathrm{DR}_{8}$ & 3.608 & 3.092 & 2.576 & 2.061 & 1.546 \\
\hline 9.0 & $\mathrm{DR}_{9}$ & 2.274 & 1.949 & 1.624 & 1.299 & 0.975 \\
\hline 10.0 & $\mathrm{DR}_{10}$ & 3.372 & 2.890 & 2.408 & 1.927 & 1.445 \\
\hline \multicolumn{2}{|c|}{ AVERAGE } & $2.91 \pm 0.61$ & $2.50 \pm 0.52$ & $2.08 \pm 0.43$ & $1.66 \pm 0.35$ & $1.25 \pm 0.26$ \\
\hline
\end{tabular}

Table 8. Computed values of annual effective dose and annual equivalent dose rate of Rebisi Town (RB)

\begin{tabular}{llllll}
\hline $\mathrm{S} / \mathrm{N}$ & $\mathrm{S} / \mathrm{Pts}$ & $\mathrm{C}_{R N}(\mathrm{PCi} / \mathrm{L})$ & $\begin{array}{l}\mathrm{C}_{R N} \\
\left(B q / \mathrm{m}^{3}\right)\end{array}$ & $\begin{array}{l}D_{R n} \\
(\mathrm{mSv} / \mathrm{y})\end{array}$ & $\begin{array}{l}\text { AEDR } \\
(\mathrm{mSv} / \mathrm{y})\end{array}$ \\
\hline 1.0 & $\mathrm{RB}_{1}$ & 0.35 & 12.9500 & 3.2671 & 0.784 \\
2.0 & $\mathrm{RB}_{2}$ & 0.19 & 7.0300 & 1.7736 & 0.426 \\
3.0 & $\mathrm{RB}_{3}$ & 0.27 & 9.9900 & 2.5204 & 0.605 \\
4.0 & $\mathrm{RB}_{4}$ & 0.16 & 5.9200 & 1.4935 & 0.358 \\
5.0 & $\mathrm{RB}_{5}$ & 0.31 & 11.4700 & 2.8937 & 0.694 \\
6.0 & $\mathrm{RB}_{6}$ & 0.12 & 4.4400 & 1.1202 & 0.269 \\
7.0 & $\mathrm{RB}_{7}$ & 0.24 & 8.8800 & 2.2403 & 0.538 \\
8.0 & $\mathrm{RB}_{8}$ & 0.33 & 12.2100 & 3.0804 & 0.739 \\
9.0 & $\mathrm{RB}_{9}$ & 0.11 & 4.0700 & 1.0268 & 0.246 \\
10.0 & $\mathrm{RB}_{10}$ & 0.23 & 8.5100 & 2.1470 & 0.515 \\
\hline AVERAGE & $0.23 \pm 0.03$ & $8.55 \pm 1.00$ & $2.16 \pm 0.25$ & $0.52 \pm 0.06$ \\
\hline
\end{tabular}

Table 9. Computed values of excess lifetime cancer risk for ages of $70(\mathrm{Std}) 60,50,40$ and $30 \mathrm{yrs}$ of Rebisi Town (RB)

\begin{tabular}{lllllll}
\hline $\mathrm{S} / \mathrm{N}$ & $\mathrm{S} / \mathrm{Pts}$ & $\begin{array}{l}\mathrm{ELCR} \times 10^{-3} \\
70 \mathrm{yrs}(\mathrm{Std})\end{array}$ & $\begin{array}{l}\mathrm{ELCR} \times 10^{-3} \\
60 \mathrm{yrs}\end{array}$ & $\begin{array}{l}\text { ELCR } \times 10^{-3} \\
50 \mathrm{yrs}\end{array}$ & $\begin{array}{l}\text { ELCR } \times 10^{-3} \\
40 \mathrm{yrs}\end{array}$ & $\begin{array}{l}\text { ELCR } \times 10^{-3} \\
30 \mathrm{yrs}\end{array}$ \\
\hline 1.0 & $\mathrm{RB}_{1}$ & 2.745 & 2.352 & 1.960 & 1.568 & 1.176 \\
2.0 & $\mathrm{RB}_{2}$ & 1.490 & 1.277 & 1.064 & 0.851 & 0.638 \\
3.0 & $\mathrm{RB}_{3}$ & 2.118 & 1.815 & 1.512 & 1.210 & 0.907 \\
4.0 & $\mathrm{RB}_{4}$ & 1.255 & 1.075 & 0.896 & 0.717 & 0.538 \\
5.0 & $\mathrm{RB}_{5}$ & 2.431 & 2.083 & 1.736 & 1.389 & 1.042 \\
6.0 & $\mathrm{RB}_{6}$ & 0.941 & 0.807 & 0.672 & 0.538 & 0.403 \\
7.0 & $\mathrm{RB}_{7}$ & 1.882 & 1.613 & 1.344 & 1.075 & 0.807 \\
8.0 & $\mathrm{RB}_{8}$ & 2.588 & 2.218 & 1.848 & 1.479 & 1.109 \\
9.0 & $\mathrm{RB}_{1}$ & 0.863 & 0.739 & 0.616 & 0.493 & 0.370 \\
10.0 & $\mathrm{RB}_{10}$ & 1.804 & 1.546 & 1.288 & 1.031 & 0.773 \\
\hline AVERAGE & $1.82 \pm 0.21$ & $1.55 \pm 0.18$ & $1.29 \pm 0.15$ & $1.04 \pm 0.12$ & $0.78 \pm 0.09$ \\
\hline
\end{tabular}

$[7,11]$ is given as:

$$
\operatorname{DRn}(m S v / y)=C R n \cdot D \cdot H \cdot T . F
$$

where $C R n=$ the the measured concentration of indoor radon222 in $\mathrm{Bqm}^{-3}, F=$ Radon-222 equilibrium indoor factor of (0.4), $T=$ the indoor- 222 occupancy time $7000 \mathrm{hr},(0.8 \times 24 \mathrm{hr} \times$ $365), H=$ indoor radon-222 occupancy factor (0.4), and $D=$ dose conversion factor $\left(9.0 \times 10^{-6} \mathrm{mSv} / \mathrm{hr}\right.$ per $\left.\mathrm{Bqm}^{-3}\right)$.

\subsubsection{Annual Effective Dose Rate (AEDR) from Radon Con- centration}

The annual dose rate was calculated by applying a tissue and radiation weighting factor $[12,13]$. The inhalation dose equation is given as:

$$
\operatorname{AEDR}(m S v / y)=D R n . W R \cdot W T
$$

where $D R N=$ indoor radon concentration, $W R=$ radiation 

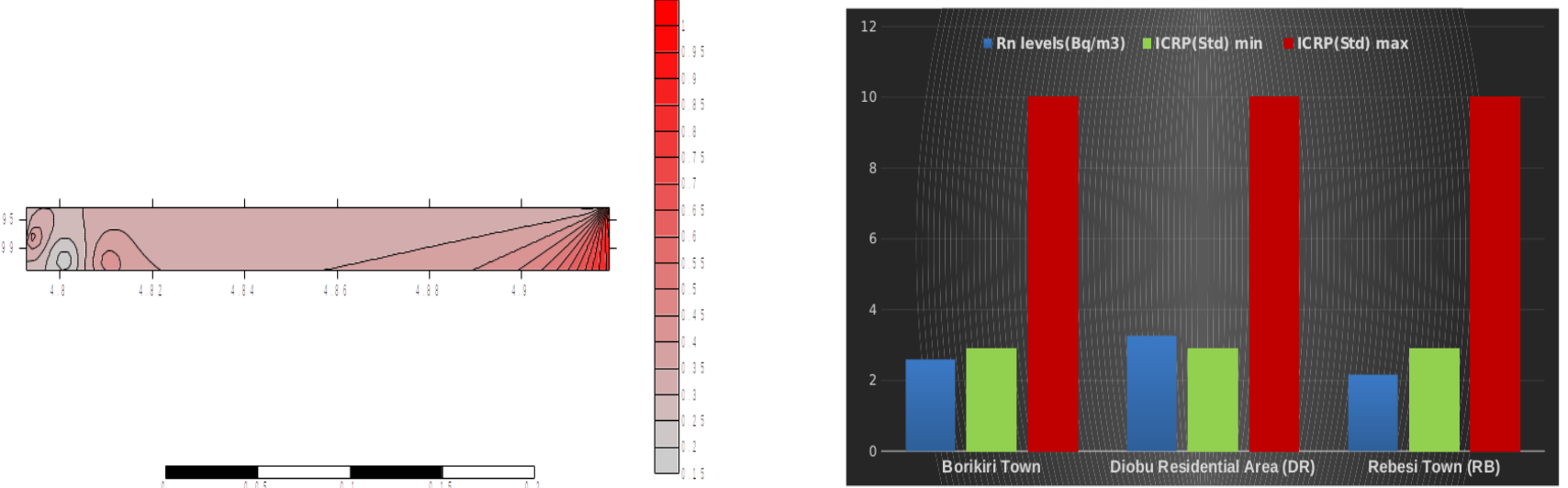

Figure 3. Contour map of Diobu Residential Area (DR)

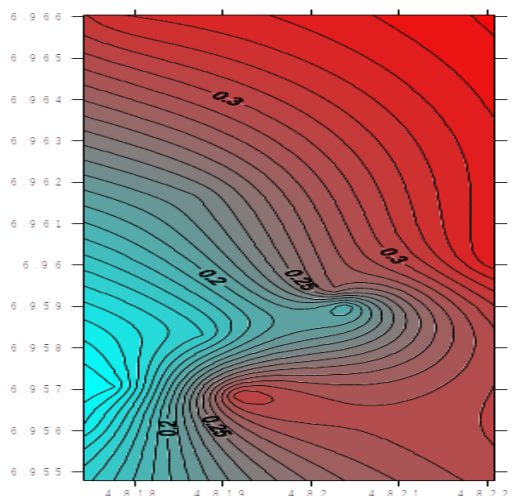

Figure 6. Annual Absorbed Dose of sample locations and ICRP Standard

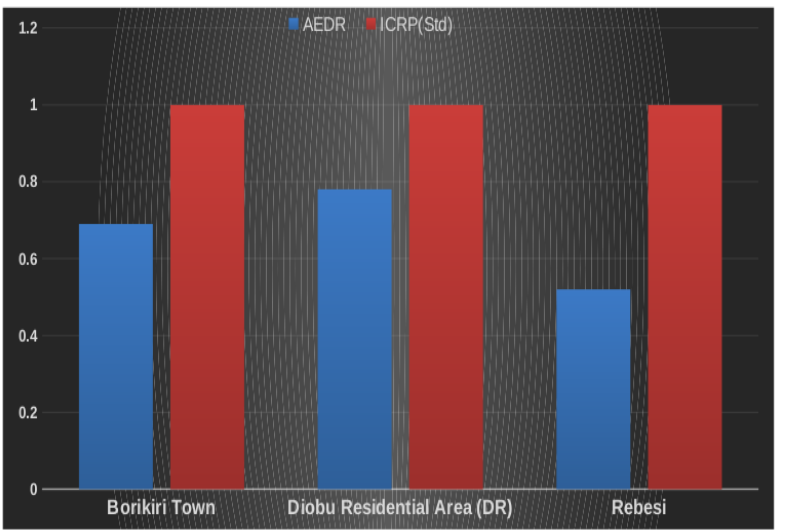

Figure 4. Contour map of Rebesi Village (RB)

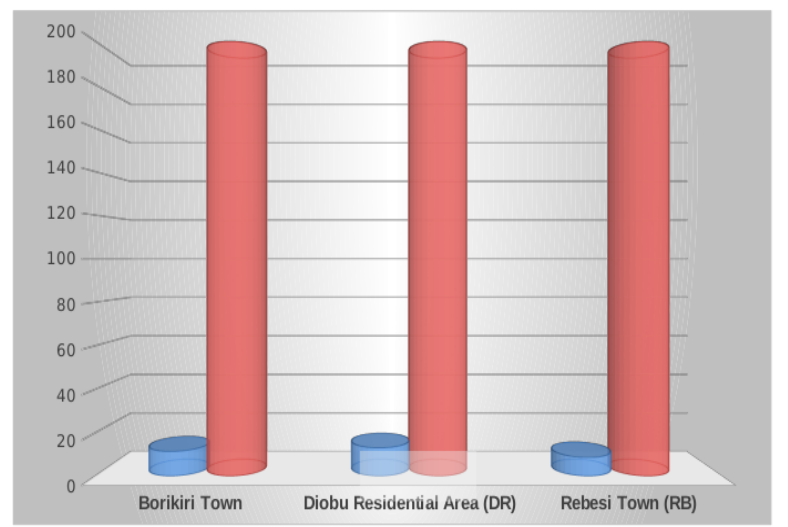

Figure 7. Annual Effective Dose Rate (AEDR) and ICRP Standard

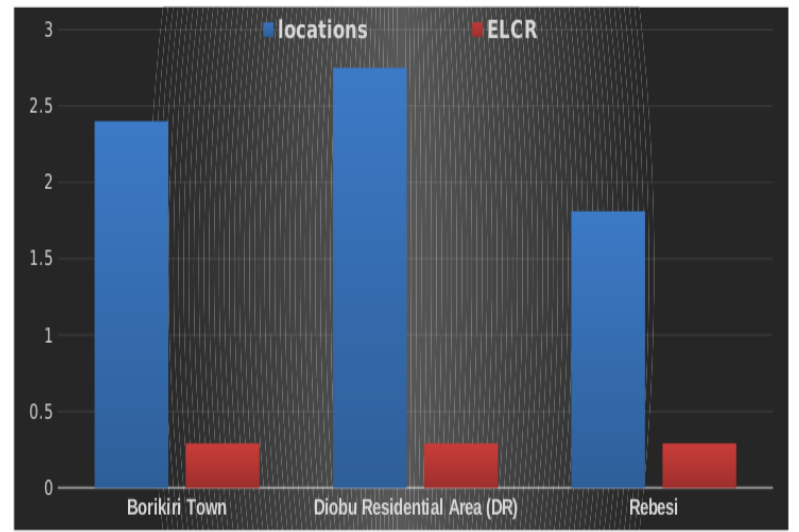

Figure 5. Average Indoor Radon Level of sample locations and ICPR reference 1

Figure 8. Excess Life Time Cancer Risk (ELCR) of sample locations with World Standard

weighting factor for alpha particles (20), and $W T=$ tissue weighting factor for the lung $(0.12)$.

\subsubsection{Excess Life Time Cancer Risk(ELCR) from Radon Con- centration}

The excess life time cancer risks (ELCR) is the potential carcinogenic effects, from the calculation based on probability of cancer induced incidence in a population. It indicates the

chances of contracting a cancer from the exposure from radiation or toxic chemical substances for a specific life time. According to [14] the excess life time risk was calculated from the equation:

$$
E C L R=A E D R \times D L \times R F
$$

where $A E D R=$ Annul effective dose rate, $D L=$ Average duration of life (70 years), $R F=$ Risk Factor $(0.05)$ 


\section{Statistical Analysis}

The mean values and standard deviation for the radon concentrations was computed using equations (4) and (5). This can also be computed with the aid of a histogram and contour maps showing the spatial distribution of radon concentration and its health risk parameters which will also be plotted with the aid of statistical package for social sciences (SPSS) version (22) and surfer- 8 contour map software.

$$
\begin{aligned}
& \mu_{x}=\frac{\sum_{i}^{n} x}{n} \quad \text { (Mean value) } \\
& \text { S.D. }=\sqrt{\frac{\sum_{i}^{n}\left(x_{i} \mu_{x}\right)^{2}}{n-1}}
\end{aligned}
$$

\section{Results and Discussion}

Indoor radon concentration and their geographical coordinates for the three Borikiri, Diobu and Rebisi are presented in Tables 1, 2 and 3, respectively. Tables $4-9$ represent the computed values of annual effective dose and annual equivalent dose rate and the values of excess lifetime cancer risk for ages of: 70(Std), 60, 50, 40 and 30 yrs of Borikiri , Diobu and Rebesi towns respectively.

From the results obtained, the indoor radon concentration level for the towns as $30.7100-19.9800 \mathrm{~Bq} / \mathrm{m}^{3}, 37.7400-$ $5.9200 \mathrm{~Bq} / \mathrm{m}^{3}$ and $12.9500-4.0700$ respectively. The annual absorbed dose for the thre communities varied as 7.7478-1.1202 $\mathrm{mSv} / \mathrm{yr}, 9.5214-1.1494 \mathrm{mSv} / \mathrm{yr}$ and $3.2671-1.0268 \mathrm{mSv} / \mathrm{yr}$ with averages of $0.69 \pm 0.16 \mathrm{~m} \mathrm{~Sv} / \mathrm{yr}, 3.26 \pm 0.73 \mathrm{mSv} / \mathrm{yr}$ and $2.16 \pm 0.25 \mathrm{mSv} / \mathrm{yr}$. The annual equivalent dose rate ranged from $0.829-0.336 \mathrm{mSv} / \mathrm{yr}, 0.694-0.359 \mathrm{mSv} / \mathrm{yr}, 0.784-0.269$ $\mathrm{mSv} / \mathrm{yr}$ with mean values of $0.69 \pm 0.16 \mathrm{mSv} / \mathrm{yr}, 0.78 \pm 0.78$ $\mathrm{mSv} / \mathrm{yr}, 0.52 \pm 0.06 \mathrm{mSv} / \mathrm{yr}$. The excess life time cancer risk calculated for seventy years (70yrs) varied from $6.510-0.941$, $8.000-1.725,2.745-0.863$ with mean values of $2.45 \pm 1.71$, $2.91 \pm 0.61,1.82 \pm 0.21$. The measured values of the indoor radon concentration and the calculated values of the annual absorbed dose, annual equivalent dose rate are all lower than the set standard by the International Commission on Radiological Protection (ICRP). The calculated values of the excess life time cancer risk calculated for life time of $70 \mathrm{yrs}, 60 \mathrm{yrs}, 50 \mathrm{yrs}, 40 \mathrm{yrs}$ and 30 yrs are all higher than the set limit by International Commission on Radiological Protection (ICRP). However, there are no observed cases of lung cancer epidemic in these areas. The contour maps of the study area are plotted in Figures 2, 3, and 4, respectively. The red colour on the contour map represents area of high radon concentration while the green colour represents area of very low radon distribution. Also, the values of the indoor concentration levels, annual absorbed dose, annual equivalent dose rate and the excess life time cancer risk are plotted in the bar-chart of Figures 5, 6, 7, and 8, respectively.

From the result obtained, there is no appreciable difference between the different study areas, this is due to the similarities in the building design, materials used in the building, ventilation rate of the living houses and the life style of the people. These results obtained are comparable to the indoor radon concentration level for different types of buildings in covenant university, Nigeria by [15]. The mean radon concentration for the three different building types Glass, Brick house and Basement house were $14.96 \mathrm{Bqm}^{-3}, 10.74 \mathrm{Bqm}^{-3}$, and $144,6 \mathrm{Bqm}^{-3}$, respectively. However, the concentration level for the basement house was higher than the recommended value by the International Commission on Radiological Protection (ICRP). Also, the indoor radon level concentration measured by [16] in Obasfemi Awolowo University Ile-Ife are similar to the indoor radon level measured in Borikiri, (BT), Diobu (DR) and Rebisi (RB) towns. The radon level obtained from the offices varied from $0.0-5.3 \mathrm{Bqm}^{-3}$. The average value obtained was $0.9 \mathrm{pCi} / \mathrm{L}$.

Similarly, the health risk parameters, the annual absorbed dose, the annual equivalent dose rate and the excess life time cancer risk agreed with the calculated values in Borikiri, Diobu and Rebisi towns. The indoor radon level concentration measured at Borikiri, Diobu and Rebisi towns are also comparable to the work done earlier by [17] in Okrika local government area in Rivers state, Nigeria. The average concentration level was $19.36 \pm \mathrm{Bqm}-3$ for mud house, while the overall average indoor radon concentration was $11.37 \pm 3.28 \mathrm{Bqm}^{-3}$ for Okirika local government.

Also, the overall average of the annual absorb dose was $3.36 \pm 0.26 \mathrm{mSv} / \mathrm{yr}$, the mean annual effective dose rate was $0.15 \pm 0.42 \mathrm{mSv} / \mathrm{yr}$, These values are all lower than the ICRP set standard. However, the overall excess life time cancer risk calculate from the indoor radon concentration level was $(0.52 \pm$ $0.15) \times 10^{-3}$ and all above the world standard of $0.029 \times 10^{-3}$.

\section{Conclusion}

The analysis of indoor radon concentration level and its health risk parameters has been done in Borikiri, Diobu and Trans-Amadi towns in Port Harcourt, Rivers State, Nigeria using the Corentium Arthings radon digital detector. The concentration levels measured for the three towns were all below the action level of $200-600 \mathrm{~Bq} / \mathrm{m}^{3}$ given by the International Commission on Radiological Protection (ICRP). However, the excess life time cancer risk for the three towns were all higher than the world average. It was also observed that there was no appreciable difference in the indoor radon concentration level between the three towns Borikiri, Diobu and Rebisi respectively. This is because of the similarities in the building styles, materials used for the buildings, the life style of the people and the ventilation methods.

\section{References}

[1] T.J Ojo and I. Ajayi, "Outdoor Radon Concentration in the Township of Ado- Ekiti Nigeria”, Journal of Atmospheric Pollution 3 (2015) 21.

[2] IARC CD-ROM, Globocan 1: Cancer Incidence and Mortality Worldwide in 1990, Lyon, International Atomic Energy Agency Press (1998). 
[3] WHO. WHO Handbook on indoor radon: "A public health perspective" World Health Organization, Geneva, (2009).

[4] WHO "Handbook on Indoor Radon" World Health Organisation (2009).

[5] UNSCEAR "Effects and risks of ionizing radiations. New York: United Nations Scientific Committee on the Effects of Atomic Radiation" (2000).

[6] NCRP (U.S. National Council on Radiation Protection and Measurement) "Measurement of "Radon and Radon Daughters in air", NCRP Report No97, ISBN 0-913392-97-9, Bethesda (1988).

[7] ICRP "Protection against Radon-222 at Home and at Work," International Commission on Radiological Protection Publication 65: Ann Icrp 23 (1993).

[8] UNSCEAR, "Sources and Effects of Ionizing Radiation", United Nations Scientific Committee on the Effects of Atomic Radiation (1993).

[9] A.W.K. Yeun, The "As Low As Reasonably Achievable" (ALARA) principle: a brief historical overview and a bibliometric analysis of the most cited publications Radioprotection (2019).

[10] W. S. Kent, and S. E. William "Geochemical and Ray Characterization of Pennsylvanian Black Shales: Implications for Elevated Home Radon Levels in Vanderburgh County, Indiana", Journal of Environmental Radioactivity 148 (2015) 162.

[11] UNSCEAR. Sources and Effects of Ionizing Radiation. Volume II: Effects. UNSCEAR (2000) Report. United Nations Scientific Committee on the Effects of Atomic Radiation, Report to the General Assembly, with scientific annexes. United Nations sales publication E.00.IX.4. United Nations, New York, (2000).

[12] ICRP "Recommendations of the International Commission on Radiological Protection” ICRP Publication 60 Ann. Icrp 21 (1991) 3.

[13] ICRP "Lung Cancer Risk from Radon and Progeny, statement on Radon" International Commission on radiological Protection Publication115.Annal ICRP 40 (2010).

[14] M. R. Usikalu, C. A. Onumejor, J. A. Achuka, A. Akinpelu, M. Omeje and T. A. Adagunodo" Monitoring of radon concentration for different building types in Covenant University, Nigeria," Cogent Engineering 7 (2020) 1759396

[15] S. A. Sokari, "Estimation of Radiation Risks Associated with Radon within Residential Buildings in Okrika, Rivers State, Nigeria”, Asian Journal of Physical and Chemical Sciences 6 (2018) 1.

[16] O. T. Afolabi, T.E. Deborah, B. Bosun, A. F Benjamin., E. T. James and B. O. Babakayode 'Radon level in a Nigerian University Campus'. BMC Res Notes 8 (2015) 677

[17] S. A. Sokari, "Estimation of Radiation Risks Associated with Radon within Residential Buildings in Okrika, Rivers State, Nigeria", Asian Journal of Physical and Chemical Sciences 6 (2018) 12. 